\title{
Metabolic imaging of tumor for diagnosis and response for therapy
}

Elena Zagaynova, Marina Shirmanova, Maria Lukina,
Varvara Dudenkova, Nadezgda Ignatova, et al.

Elena Zagaynova, Marina Shirmanova, Maria Lukina, Varvara Dudenkova, Nadezgda Ignatova, Vadim Elagin, Irena Shlivko, Vladislav Scheslavsky, Natalia Orlinskay, "Metabolic imaging of tumor for diagnosis and response for therapy," Proc. SPIE 10498, Multiphoton Microscopy in the Biomedical Sciences XVIII, 1049804 (23 February 2018); doi: 10.1117/12.2295127

SPIE. Event: SPIE BiOS, 2018, San Francisco, California, United States 


\title{
Metabolic imaging of tumor for diagnosis and response for
} therapy

\author{
Elena Zagaynova*a ${ }^{* a}$ Marina Shirmanova ${ }^{\mathrm{a}}$, Maria Lukina ${ }^{\mathrm{a}, \mathrm{b}}$, Varvara Dudenkova ${ }^{\mathrm{a}, \mathrm{b}}$, Nadezgda \\ Ignatova $^{\mathrm{a}}$, Vadim Elagin ${ }^{\mathrm{a}}$, Irena Shlivko ${ }^{\mathrm{c}}$, Vladislav Scheslavsky ${ }^{\mathrm{d}}$, Natalia Orlinskay ${ }^{\mathrm{c}}$ \\ ${ }^{a}$ Nizhny Novgorod State Medical Academy, 10/1 Minin and Pozharsky Sq., Nizhny \\ Novgorod, Russian Federation, 603950; 'bobachevsky State University of Nizhny Novgorod, \\ 23 Gagarin Aveue, Nizhny Novgorod, Russian Federation, 603950; ' Nizhny Novgorod \\ Research Institute of Traumatology and Orthopedics, 18 Verhne-Voljskaya naberejnaya,

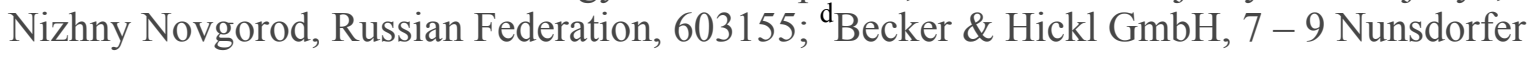 \\ Ring, Berlin, Germany 12277
}

\begin{abstract}
Nonlinear optical microscopy combined with fluorescence lifetime imaging is a non-invasive imaging technique, based on the study of fluorescence decay times of naturally occurring fluorescent molecules, enabling a noninvasive investigation of the biological tissue with subcellular resolution. Cancer exhibits altered cellular metabolism, which affects the autofluorescence of metabolic cofactors $\mathrm{NAD}(\mathrm{P}) \mathrm{H}$ and FAD. In this study features of tumor metabolism in different systems of organization (from cell culture to patient lesion) was showed. The observed differences in the relative contributions of free $\mathrm{NAD}(\mathrm{P}) \mathrm{H}$ and FAD testify to an increased a glycolytic metabolism in cancer cells compare to fibroblasts. In 3D spheroids, the cells of the proliferating zone had greater a1 and lower tm values than the cells of the quiescent zone, which likely is a consequence of their higher glycolytic rate. During the growth of colorectal cancer in the experimental mouse model, the contribution of the free component of $\mathrm{NAD}(\mathrm{P}) \mathrm{H}$ was increased. Dysplastic nevus and melanoma is characterized by raised contribution of free NADH compare to healthy skin. Therefore, melanoma cells had very short value of $\tau 1$.
\end{abstract}

\section{INTRODUCTION}

Highly proliferative cancer cells have greater needs for energy and building blocks for the synthesis of macromolecules (nucleotides, protein, and lipids) in order to duplicate genome and biomass $[1,2]$. The best characterized metabolic feature observed in tumor cells is the Warburg effect, which is a prevalence of glycolysis over oxidative metabolism, even under normal oxygen concentrations [3]. At the same time, slow-cycling cancer cells use oxidative phosphorylation (OXPHOS) and a mitochondrial supply of ATP [4]. There is a hypothesis that the lowering of oxidative metabolism can promote multiple drug resistance and help the cells to escape from apoptosis [5]. Furthermore, it has been determined that tumors are extremely metabolically heterogeneous, and metabolic phenotypes may vary from one cell to another [6]. Besides, some cancer cells can reversibly switch between glycolysis and oxidative metabolism, depending on the environmental conditions [5]. Although aerobic glycolysis is often found in malignant tumors, OXPHOS still contributes much to energy production in cancers and play an active role in cancer metabolism [6,7]. It is suggested that cancer metabolism can serve as an indicator of efficiency of anticancer treatment and be used for better understanding the mechanisms of action of anticancer drugs [8-10]. Multiphoton fluorescence lifetime imaging (FLIM) is a promising non-invasive technique for a large number of biological applications. Among the FLIM techniques, Time Correlated Single Photon Counting (TCSPC) FLIM delivers the highest time resolution and the best lifetime accuracy or photon efficiency [11]. The use of the TCSPC FLIM method has been widely demonstrated in oncology for not only basic research on cell cultures and animal tumor models but also for clinical imaging of tissue samples from patients [12]. FLIM imaging techniques are divided into microscopic systems with cell resolution for cell culture analysis in 2D and 3D conditions (laser scanning microscopy with FLIM module), a live mouse and patient imaging system: with cell resolution (multiphoton tomography), the whole body (macroscanning system) and systems based on fibers for measuring metabolic cofactors inside tissues (fiber system) [13]. Combination of different living systems, such as cancer cell cultures, 3D spheroids and organoids, living tumor bearing mice and

Multiphoton Microscopy in the Biomedical Sciences XVIII, edited by Ammasi Periasamy,

Peter T. C. So, Karsten König, Xiaoliang S. Xie, Proc. of SPIE Vol. 10498, 1049804

(C) 2018 SPIE · CCC code: 1605-7422/18/\$18 · doi: 10.1117/12.2295127 
even patients with FLIM imaging permit to solve some fundamental and practical tasks. For example, in cell culture we can investigate some fundamental process in cancer development and discover the new mechanisms of drug response, open new check points for tumor treatment. In 3D spheroids it is possible visualize tumor heterogeneity and specific features of cancer cells response in dependence of Oxygen and nutrition presence. Also, this system can be applied for creating short time 3D cell cultures, like organoids, from fresh patients tumor species. This approach can help validate the drug activity a special for this patient [14, 15]. In vivo tumor models in mice can be used for analyzing of metabolic differences during tumor growth, switching metabolism under drug treatment and discovering new combination of treatment with influence on metabolic chain. Translation metabolic imaging to clinical practice helps doctors in the process of differential diagnosis of clinical looks similar pathological lesions, for example, cancer and precancer lesions in oral cavity [16], nonmelanoma and melanoma lesions [17-19]. The aim of present work was to show different living systems and FLIM modalities for investigation of tumor metabolism as in physiological conditions as in drug response.

\section{MATERIALS AND METHODS}

\subsection{Cell culture and $3 \mathrm{D}$ spheroids}

The CT26 (murine colon carcinoma) cells were grown in DMEM supplemented with $10 \%$ fetal bovine serum (FBS), $2 \mathrm{mM}$ glutamine, $10 \mu / \mathrm{mL}$ penicillin and $10 \mathrm{mg} / \mathrm{mL}$ streptomycin. The cells were incubated at $37^{\circ} \mathrm{C}, 5 \%$ $\mathrm{CO} 2$, and $80 \%$ relative humidity and subcultivated three times a week.

For fluorescence microscopy the cells were seeded $(1 \times 105$ in $2 \mathrm{~mL})$ in glass-bottomed $35 \mathrm{~mm}$ FluoroDishes and incubated overnight $\left(37^{\circ} \mathrm{C}, 5 \% \mathrm{CO} 2\right)$. Then the cells were washed with PBS and placed in DMEM life medium without phenol red, for imaging.

For 3D culturing, HeLa cells (human cervical cancer) were seeded into 96-well ultra-low attachment round bottom plates at a concentration of 100 cells in $200 \mu \mathrm{L}$ of complete medium per well and incubated at $37^{\circ} \mathrm{C}, 5 \%$ $\mathrm{CO} 2,80 \%$ relative humidity. The medium was changed every three days. Three days after seeding, tumor spheroid formation was confirmed by microscopic examination in transmitted light. A detailed protocol for spheroid generation can be found in Ref. [20].

For fluorescence microscopy, the spheroids were transferred onto glass-bottom dishes in DMEM life medium without phenol red, and incubated for 30-60 min to allow for spheroid attachment.

\subsection{Tumor xenografts}

All animal protocols were approved by the Ethics Committee of Nizhny Novgorod State Medical Academy. Experiments were performed on female Balb/C mice purchased from the Pushchino animal nursery (Pushchino, Russia). Mice of 20-22 g body weight were inoculated subcutaneously in the left flank with CT 26 cells $(5 \times 105$ in $100 \mu \mathrm{L}$ PBS). Fluorescence microscopy was implemented in vivo on 7th, 14th and 21th days of the tumor growth. Before imaging procedure, the mice were anesthetized intramuscularly with a mixture of Zoletil (40 $\mathrm{mg} / \mathrm{kg}, 50 \mu \mathrm{L}$, Virbac SA, Carros, France) and 2\% Rometar (10 mg/kg, $10 \mu \mathrm{L}$, Spofa, Czech Republic). A skin flap over the tumor was surgically opened and the microscopic objective was placed directly on the tumor surface. After image acquisition, the animals were sacrificed by cervical dislocation and the tumors were excised for histopathology.

\subsection{Patients}

Twelve patients with pigmented skin disorders such as 1 st degree dysplastic nevi, 2nd degree dysplastic nevi and melanoma have been studied by multiphoton laser tomography. The 12 lesions were distributed in 6 primary locations, including back, arms, chest, face, legs, and abdomen. MPT examinations was performed on disorders sites as well as on neighboring healthy skin.

\subsection{FLIM imaging of NAD(P)H and FAD}

$\mathrm{NAD}(\mathrm{P}) \mathrm{H}$ and FAD fluorescence intensity and lifetime images of the monolayer cell cultures were acquired using an LSM 880 (Carl Zeiss, Germany) fluorescence confocal laser-scanning microscope equipped with an FLIM module: Simple Tau 152 TCSPC (Becker \& Hickl GmbH, Germany). A water immersion objective CApochromat 40x/1.2 NA W Korr was used for image acquisition. During image acquisition, the cells were maintained at $37^{\circ} \mathrm{C}$ and $5 \% \mathrm{CO}$. Two-photon fluorescence of $\mathrm{NAD}(\mathrm{P}) \mathrm{H}$ and $\mathrm{FAD}$ was excited with a femtosecond Ti:Sa laser (repetition rate $80 \mathrm{MHz}$, pulse duration $140 \mathrm{fs}$ ) at wavelengths of $750 \mathrm{~nm}$ or $900 \mathrm{~nm}$ and 
registered in the ranges $455-500 \mathrm{~nm}$ or $500-550 \mathrm{~nm}$, respectively. The average power applied to the samples was $\sim 6 \mathrm{~mW}$. The approximate rate of photon counting was $1-2 \times 10^{5}$ photons/second. Image collection time was 60 seconds.

For two-photon fluorescence microscopy and FLIM of the tumor spheroids, tumor in vivo and patients an MPTflex (JenLab GmbH, Germany) multiphoton tomograph, equipped with a tunable $80 \mathrm{MHz}, 200 \mathrm{fs}$ MaiTai Ti:Sa laser and a TCSPC-based FLIM module (Becker \& Hickl GmbH, Germany), was used. The images were acquired through a 40x/1.3 NA oil immersion EC Plan-Neofluar objective. Wavelengths of $750 \mathrm{~nm}$ and $900 \mathrm{~nm}$ were used for excitation of the $\mathrm{NAD}(\mathrm{P}) \mathrm{H}$ and FAD, respectively. Emissions were detected in the range 410-660 $\mathrm{nm}$ using a fixed pre-fitted emission filter. The average power applied to the sample was $\sim 10 \mathrm{~mW}$. Image collection time was 10 seconds. Images were acquired from a depth of $\sim 20 \mu \mathrm{m}$.

To calculate the optical redox ratio, the fluorescence intensity of FAD was divided by the fluorescence intensity of $\mathrm{NAD}(\mathrm{P}) \mathrm{H}$ at each pixel (ImageJ 1.39p software, USA). The background signal calculated for a cell-free area of the plate was subtracted.

The fluorescence lifetime decay curves of $\mathrm{NAD}(\mathrm{P}) \mathrm{H}$ and $\mathrm{FAD}$ were fitted to a double-exponential decay model, and the short and long lifetime components ( $\mathrm{t} 1$ and $\mathrm{t} 2$, respectively), the mean fluorescence lifetime $(\mathrm{tm}=(\mathrm{a} 1$. $\tau 1+\mathrm{a} 2 \cdot \tau 2) /(\mathrm{a} 1+\mathrm{a} 2))$ and the relative amplitudes of the lifetime components (a1 and $\mathrm{a} 2$, where $\mathrm{a} 1+\mathrm{a} 2=100 \%)$ were estimated. The goodness of the fit, the $\chi^{2}$ value, was $0.8-1.2$. In a first approximation, for NAD(P)H the first (short, t1) component is attributed to its free, and the second (long, t2) component - to its protein-bound state. For $\mathrm{FAD}, \mathrm{t} 1$ is attributed to its protein-bound and $\mathrm{t} 2$ - to its free state. SPCImage software (Becker \& Hickl GmbH, Germany) was used to analyze the FLIM data.

The redox ratio and FLIM measurements were performed in the cytoplasm of the cells by selecting $\sim 40 \times 40$ pixel zones as regions of interest.

\subsection{Statistics}

The results are expressed, below, as means \pm standard deviation (SD). To calculate the statistical significance of the differences, the ANOVA with Bonferroni post-hoc test was used.

\section{RESULTS}

\subsection{Cell culture}

As expected for FLIM, the fluorescence decay curves for NAD (P)H and FAD were best fit to a doubleexponential decay model, indicating the presence of 2 distinctly different lifetimes for the free and protein-bound forms of the co-factors. The fluorescence lifetimes of the free (t1) and protein-bound (t2) NAD (P)H were measured to be $\approx 0.4$ and $\approx 2.6 \mathrm{~ns}$, respectively. For the free (t2) and protein-bound (t1) the FAD fluorescence lifetimes were $\approx 2.7$ and $\approx 0.2$ ns. Fluorescence lifetimes were the same in both the cancer cells and fibroblasts and did not change during co-culturing. In monocultures of cancer cells and fibroblasts the relative contributions of the co-factors were fairly stable throughout the 5 days of cultivation without any statistical difference for the $\mathrm{NAD}(\mathrm{P}) \mathrm{H}$ and only a slight difference for FAD (p D 0.000011). The relative contribution of free NAD(P)H (a1) in cancer cells was high than in fibroblast culture $72.18 \%$ and $66.39 \%$ ( $\mathrm{D} 0.000001$ ). The relative contribution of free FAD (a2) in these cancer cells was also higher than in fibroblasts $75 \%$ and $71.23 \%$ (p D 0.000000 ). The observed differences in the relative contributions of free $\mathrm{NAD}(\mathrm{P}) \mathrm{H}$ and FAD testify to an increased a glycolytic metabolism in cancer cells. (Fig. 1). All cells in the population displayed the described changes. 


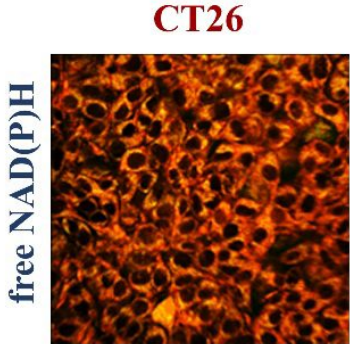

$0.41 \pm 0.03 \mathrm{~ns}$

$72.18 \pm 2.06 \%$

CT26

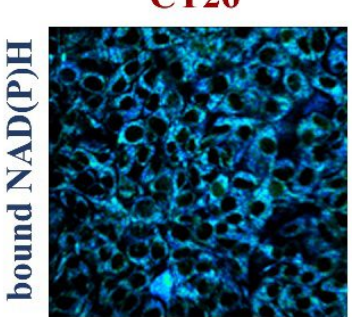

$1.88 \pm 0.08 \mathrm{~ns}$

$27.81 \pm 2.06 \%$
3T3

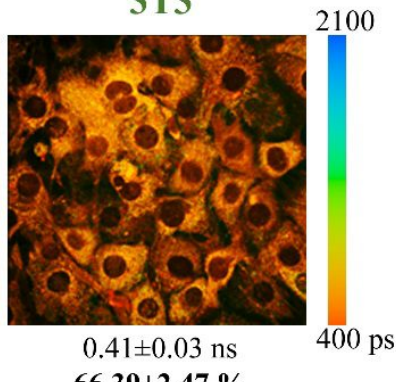

3T3

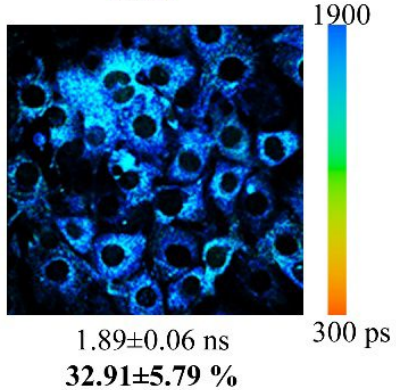

CT26

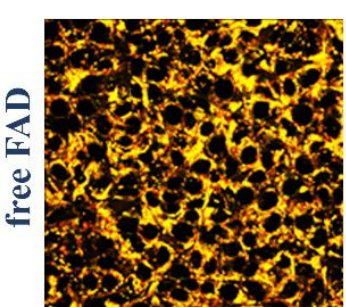

$0.24 \pm 0.01 \mathrm{~ns}$

$75.00 \pm 1.65 \%$

CT26

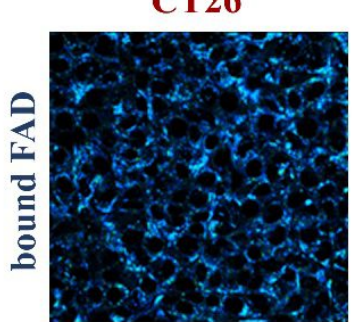

$1.58 \pm 0.08 \mathrm{~ns}$

$25.01 \pm 1.65 \%$

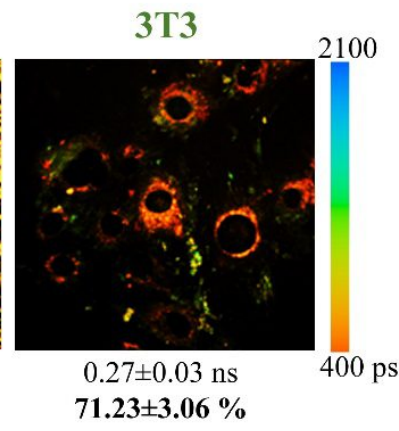

3T3

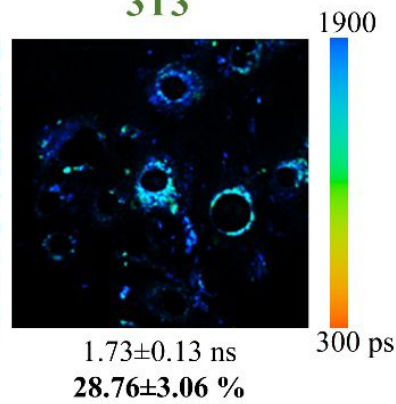

Figure 1. In vitro metabolism of cancer (CT26) and normal (3T3) murine cells.

\subsection{D spheroids}

To analyze the heterogeneity of tumor spheroids, the spheroid size, morphology and the viability of their cells were assessed during 7 days (Fig.2). By day 5 of the growth, the spheroids had formed compact multicellular spheres $\sim 350 \mu \mathrm{m}$ in diameter, where a thin outer shell of loosely packed, actively proliferating cells and an inner, more dense spheroid core could be distinguished. By day 7, the size of the spheroids had increased to $\sim 480 \mu \mathrm{m}$. The live/dead assays of spheroids revealed single dead cells during the whole period of observation from day 5 to day 7. Tumor spheroids represent intrinsically heterogeneous formations composed of cells in different proliferative and metabolic states. Spheroids with radii of $\sim 200 \mu \mathrm{m}$ and larger are known to have zones of proliferating cells on the outside and quiescent cells on the inside due to nutrient and oxygen transport limitations [21]. Therefore, we performed metabolic measurements of spheroids separately for the outer and inner layers. The focus of these experiments was on $\mathrm{NAD}(\mathrm{P}) \mathrm{H}$, because, as shown in monolayer culture, FAD fluorescence did not reflect metabolic perturbations during growth. Although, the cells in the periphery of the spheroid proliferate more actively, the redox ratio $\mathrm{FAD} / \mathrm{NAD}(\mathrm{P}) \mathrm{H}$ did not differ between outer and inner layers. However, the relative abundance of protein-free $\mathrm{NAD}(\mathrm{P}) \mathrm{H}(\mathrm{a} 1)$ and the mean fluorescence lifetime $(\mathrm{tm})$ of $\mathrm{NAD}(\mathrm{P}) \mathrm{H}$ in the outer and inner layers were intrinsically different. The cells of the proliferating zone had greater a1 and lower tm values than the cells of the quiescent zone, which likely is a consequence of their higher glycolytic rate and is consistent with the general principle of metabolic organization of tumor spheroids. No significant metabolic perturbations were seen across the spheroids during the natural growth from day 5 to day 7 (Fig. 2). 

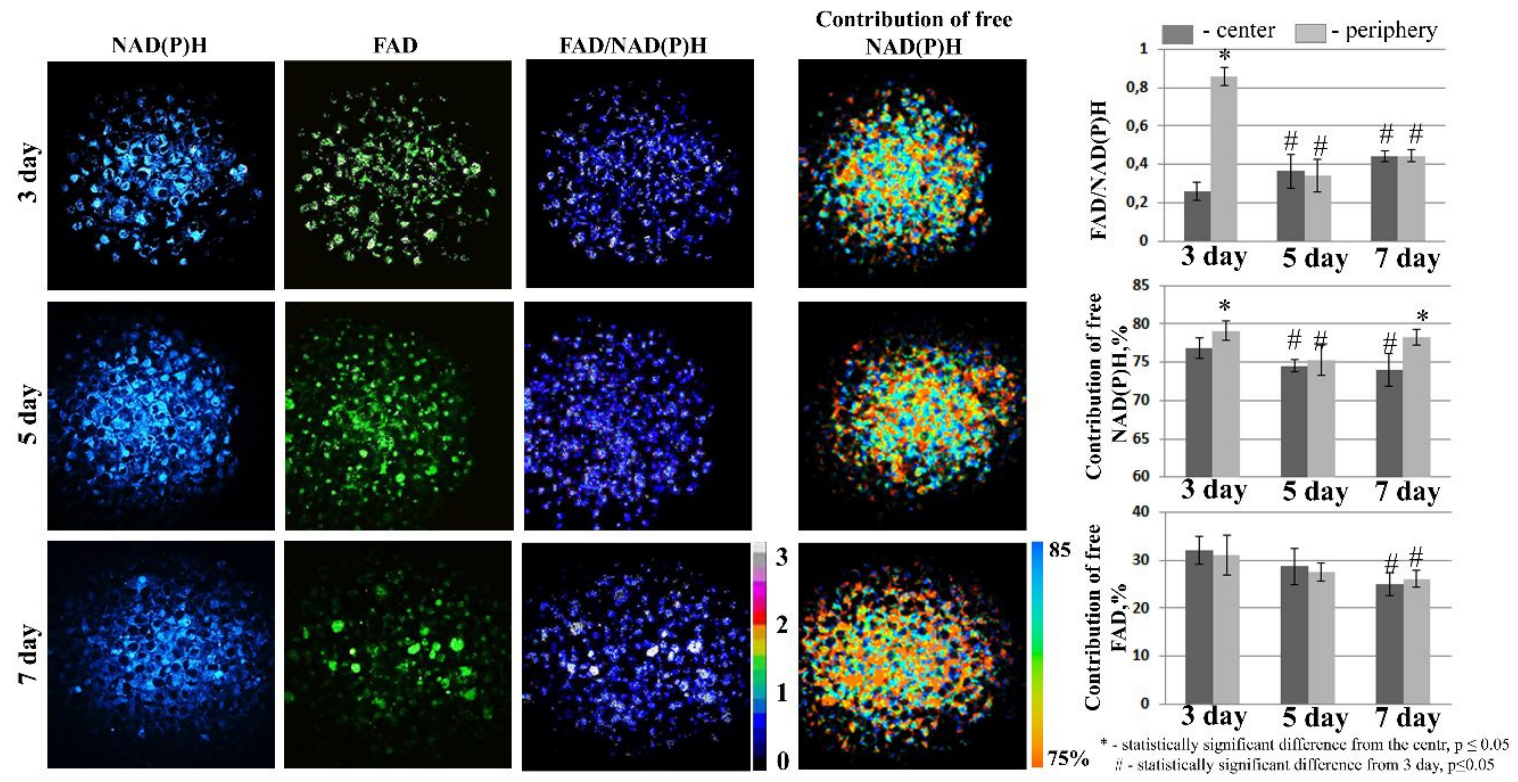

Figure 2. Heterogeneity of cancer cells (Hela Kyoto) metabolism during spheroid growth.

\subsection{In vivo mice}

Previously, it was shown that the tumor has metabolic heterogeneity, depending on the severity of the stroma. In more rich stroma (collagen, vessels) zones, more glycolytic metabolism is observed, which is reflected in an increase in the percentage contribution of the free form of NADH [22]. In this paper, we show that during the growth of colorectal cancer in the experimental mouse model, the lifetime of the free component of NADH does not change and remains approximately from 7 to 21 days at the level $\sim 0.5 \mathrm{~ns}$. But the contribution of the free component of NAD (P)H on day 7 is $82.4 \pm 1.9$, then, first falls to the 14 th day of growth to $80.1 \pm 3.1$, and then to day 21 again grows. The increase in glycolytic processes (increase in the contribution of the free component of $\mathrm{NAD}(\mathrm{P}) \mathrm{H})$ after three weeks coincides with the rapid growth and division of tumor cells, which requires a large amount of fast energy. (Fig. 3). 

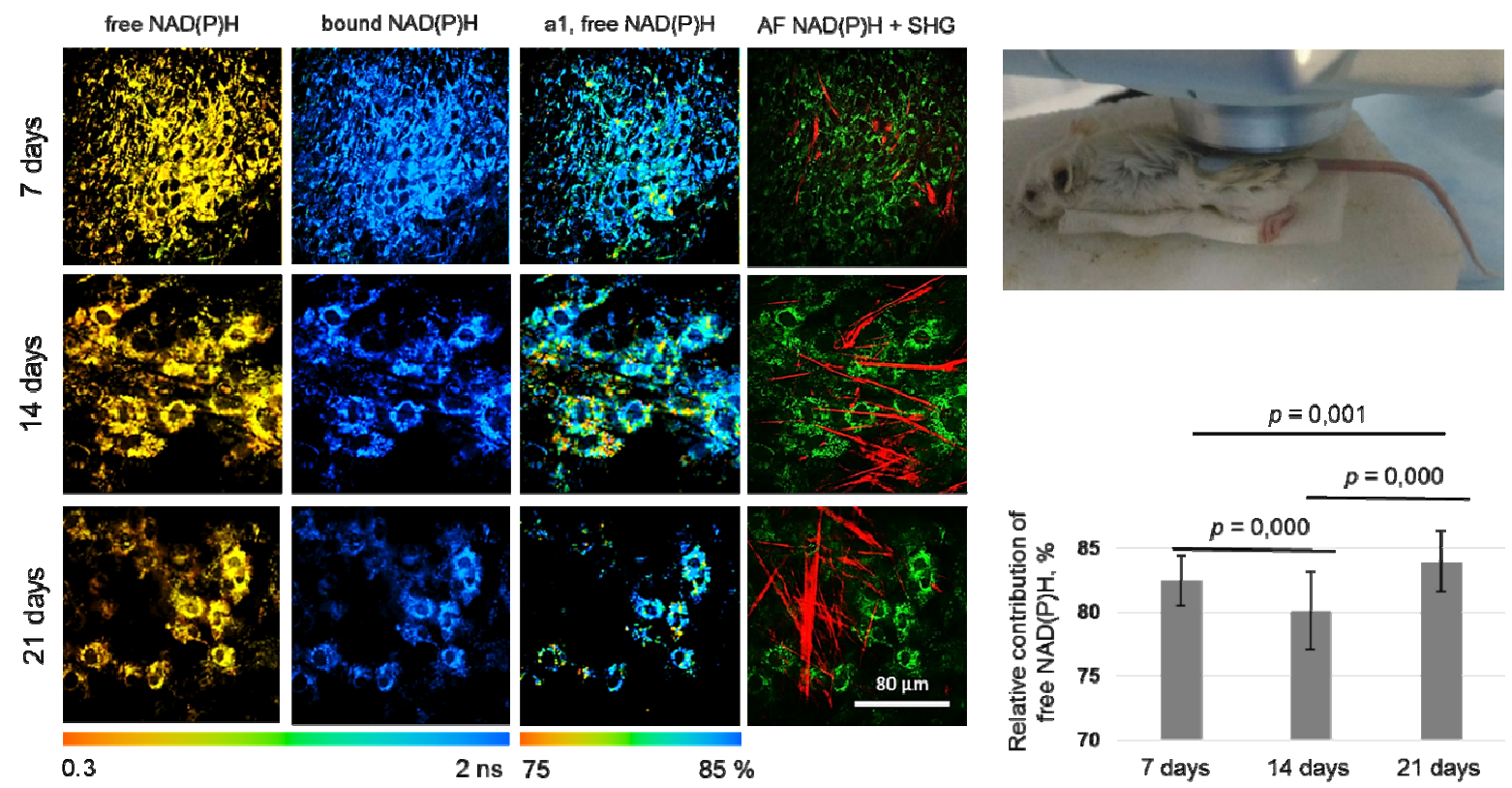

Figure 3. Changing of in vivo cancer cell metabolism during tumor growth in tumor bearing mice (CT26). NAD(P)H $\lambda$ ex $750 \mathrm{~nm}, \lambda$ em 410-660 nm; SHG $\lambda$ ex $750 \mathrm{~nm}$, $\lambda$ em $373-387 \mathrm{~nm}$.

\subsection{Patients' skin lesions}

The MPT features of healthy skin are characterized by normal morphology and architecture of cells in the epithelium layers. Pigmented keratinocytes are presented in the basal layer. Dysplastic nevi were characterized by mild cellular atypia (enlarged nuclei) and mild architectural disorder. Melanocytes were typically visualized as small and sickle-shaped cells in stratum basale and stratum spinosum. Nests of atypical melanocytes were found on the epidermal-dermal junction and on the lateral surface of the epidermal ridges. MPT images of melanoma showed the abnormal tissue architecture, the occurrence of bright luminescent cells, wide intercellular spaces, and the presence of atypical cells in all epidermis layers. Melanocytic dendrites were found in stratum granulosum. Highly pleomorphic melanocytes were detected in the epidermis, as well as in the dermis. The tissue architectural disorder was detected. It was found that the contribution of free NADH (a1) for unpigmented cells of healthy skin was $72.65 \pm 2.05 \%$ for stratum granulosum, $70.63 \pm 0.53 \%$ for stratum spinosum, and $69.74 \pm 0.56 \%$ for stratum basale. Dysplastic nevus was characterized by increased of free NADH contribution: $75 \pm 1.12 \%$ (stratum granulosum), $78.28 \pm 2.37 \%$ (stratum spinosum) and 78.27 $\pm 0.84 \%$ (stratum basale). The contribution of free NADH for melanoma was $78.83 \pm 4.03 \%$. It should be noted, that basal layer of healthy skin as well as lower epithelium layers of dysplastic nevus contained pigmented keratinocytes and melanocytes. These cells were characterized by extremely short value of $\tau 1$ closed to instrumental response function (IRF) and a1 about $90 \%$ due to high content of melanin (Fig.4). 

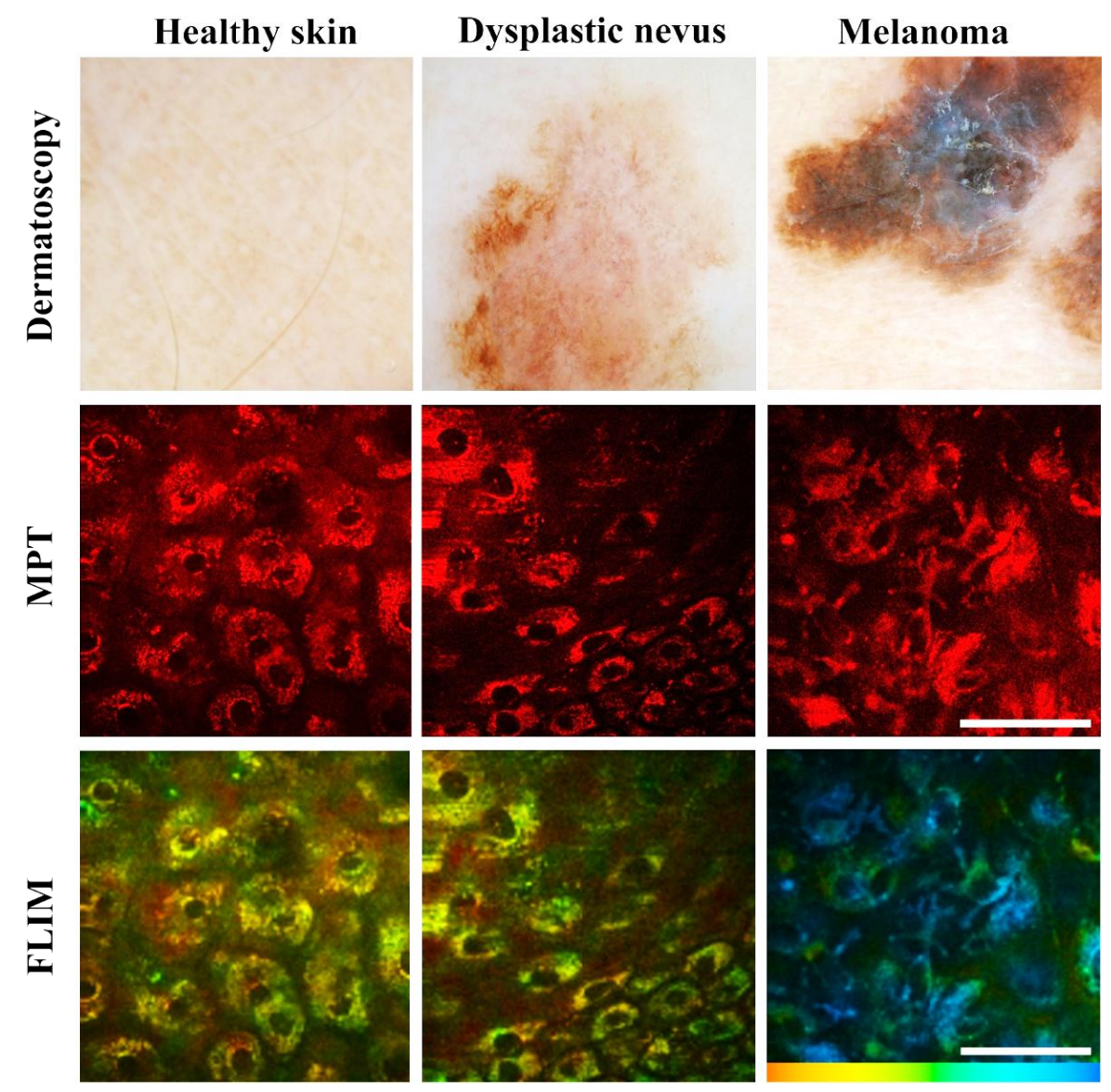

Figure 4. Dermatoscopy, Fluorescence intensity and FLIM images of healthy skin, dysplastic nevus and melanoma at depths corresponding to stratum granulosum. Scale bar, $50 \mu \mathrm{m}$. False color-coded images demonstrate contribution of short lifetime component (a1) maps. Color range: $65 \%$ (red) to $100 \%$ (blue). Scale bar: $50 \mu \mathrm{m}$.

\section{CONCLUSION}

The results of this study provide an initial set of FLIM features that are characteristic of tumor in different organization levels (from cell culture and 3D spheroids, experimental tumor-bearing mice to patients). Nonlinear optical microscopy with FLIM module was used for metabolism analysis of cancer cells in cell culture and 3D spheroids model. Multiphoton tomography with FLIM provided in vivo investigation on tumor-bearing mice and patients. Each system of organization of tumor cells allows us to solve fundamental and specific problems. On cell cultures, one can study the mechanism of action of drugs, as already known, with reference to a particular patient, and new ones, from the point of view of their influence on metabolism. On 3D spheroids, the heterogeneity of tumor cells is indicative, which can more accurately reflect the response of tumor cells to treatment. In experimental models of tumors in mice, it is possible to take into account the vital activity of cancer cells in the tumor microenvironment, which actively affects both the growth and the response of the tumor to treatment, which is reflected in the metabolic activity of the tumor cells. In the study of human skin pigmented lesions, FLIM imaging will be an indispensable tool for differential diagnosis of benign neoplasia and melanoma. The combination of cell resolution, real-time information acquisition and non-invasiveness makes the multiphoton tomography method with FLIM a unique tool for clinicians. 


\section{REFERENCES}

[1] Jang, M., Kim, S. S., Lee J., "Cancer cell metabolism: implications for therapeutic targets," Experimental \& Molecular Medicine 45, (2013).

[2] Antone, P. D., "Energy metabolism in cancer cells: How to explain the Warburg and Crabtree effects?," Medical Hypotheses 79 388-392, (2012).

[3] Cairns, R. A., Harris, I. S., Mak, T. W., "Regulation of cancer cell metabolism," Nature Reviews Cancer 11, 85-95, (2011).

[4] Marusyk, A., Polyak, K., "Tumor heterogeneity: Causes and consequences," Biochimica et Biophysica Acta (BBA) - Reviews on Cancer 1, 105-17 (2009).

[5] Diaz-Ruiz, R., Rigoulet, M., Devin, A., "The Warburg and Crabtree effects: On the origin of cancer cell energy metabolism and of yeast glucose repression," Biochimica et Biophysica Acta 1807, 568-576 (2011).

[6] Zheng, J., "Energy metabolism of cancer: Glycolysis versus oxidative phosphorylation," Oncology Letters 4, 1151- 1157 (2012).

[7] Sengupta, D., Pratx, G., "Imaging metabolic heterogeneity in cancer," Sengupta and Pratx Molecular Cancer 15(4), 1- 12 (2016).

[8] Shah, A. T., Diggins, K. E., Walsh, A. J., Irish, J. M., Skala, M. C., "In Vivo Autofluorescence Imaging of Tumor Heterogeneity in Response to Treatment," Neoplasia 17, 862-870, (2015).

[9] Marchetti, P., Guerreschi, P., Mortier, L., Kluza, J., "Integration of Mitochondrial Targeting for Molecular Cancer Therapeutics," International Journal of Cell Biology, (2015).

[10] Armitage, E. G., Southam, A. D., "Monitoring cancer prognosis, diagnosis and treatment efficacy using metabolomics and lipidomics," Metabolomics 12:146, (2016).

[11]Becker, W., "Fluorescence lifetime imaging--techniques and applications," J Microsc. 247, 119-136 (2012)

[12] Periasamy, A., Clegg, R.M., [FLIM Microscopy in Biology and Medicine], Chapman and Hall/CRC, 472 Pages (2009)

[13] Becker, W., [The bh TCSPC Handbook. 7th edition], Becker \& Hickl GmbH, 891 Pages (2017)

[14] Shah, A. T., Diggins, K. E., Walsh, A. J., Irish, J. M., Skala, M. C., "In Vivo Autofluorescence Imaging of Tumor Heterogeneity in Response to Treatment," Neoplasia 17(12), 862-870 (2015)

[15] Shah, A. T., Heaster, T. M., Skala, M. C., "Metabolic Imaging of Head and Neck Cancer Organoids," PLoS One 12(1), e0170415 (2017)

[16] Sun, Y., Phipps, J. E., Meier, J., Hatami, N., Poirier, B., Elson, D. S., Farwell, D. G., Marcu, L., "Endoscopic fluorescence lifetime imaging for in vivo intraoperative diagnosis of oral carcinoma," Microsc Microanal 19(4), 791-798 (2013)

[17] Manfredini, M., Arginelli, F., Dunsby, C., French, P., Talbot, C., König, K., Pellacani, G., Ponti, G., Seidenari, S., "High-resolution imaging of basal cell carcinoma: a comparison between multiphoton microscopy with fluorescence lifetime imaging and reflectance confocal microscopy," Skin Res Technol 19(1), e433-443 (2013)

[18] Seidenari, S., Arginelli, F., Dunsby, C., French, P. M., König, K., Magnoni, C., Talbot, C., Ponti, G., "Multiphoton laser tomography and fluorescence lifetime imaging of melanoma: morphologic features and quantitative data for sensitive and specific non-invasive diagnostics," PLoS One 8(7), e70682 (2013)

[19] Balu, M., Kelly, K. M., Zachary, C. B., Harris, R. M., Krasieva, T. B., König, K., Durkin, A. J., Tromberg, B. J., "Distinguishing between benign and malignant melanocytic nevi by in vivo multiphoton microscopy," Cancer Res 74(10), 2688-2697 (2014)

[20] Shirmanova, M. V., Shimolina, L. E., Lukina, M. M., Zagaynova, E. V., Kuimova, M. K., "Live Cell Imaging of Viscosity in 3D Tumour Cell Models," In: Dmitriev R. (Eds) Multi-Parametric Live Cell Microscopy of 3D Tissue Models. Advances in Experimental Medicine and Biology, 1035. Springer, Cham (2017)

[21]Zanon,i M., Piccinini, F., Arienti, C., Zamagni, A., Santi, S., Polico, R., Bevilacqua, A., Tesei, A., "3D tumor spheroid models for in vitro therapeutic screening: A systematic approach to enhance the biological relevance of data obtained," Scientific Reports 6, 19103. (2016)

[22] Lukina, M., Shirmanova, M., Dudenkova, V., Druzhkova, I., Shumilova, A., Zagaynova, E., "Analysis of energy metabolism of HeLa cancer cells in vitro and in vivo using fluorescence lifetime microscopy," Proc. of SPIE,9887, 98872S-2, (2016) 